\title{
Singlet extensions of the MSSM in the quiver landscape
}

\author{
Mirjam Cvetič, ${ }^{a, b, d}$ James Halverson ${ }^{a, d}$ and Paul Langacker ${ }^{c, d}$ \\ ${ }^{a}$ Department of Physics and Astronomy, University of Pennsylvania, \\ Philadelphia, PA 19104-6396, U.S.A. \\ ${ }^{b}$ Center for Applied Mathematics and Theoretical Physics, \\ University of Maribor, Maribor, Slovenia \\ ${ }^{c}$ School of Natural Science, Institute for Advanced Study, \\ Einstein Drive, Princeton, NJ 08540, U.S.A. \\ ${ }^{d}$ Kavli Institute for Theoretical Physics, Kohn Hall, \\ UCSB, Santa Barbara, CA 93106, U.S.A. \\ E-mail: cvetic@cvetic.hep.upenn.edu, jhal@physics.upenn.edu, \\ pgl@ias.edu
}

AbSTRACT: We map out possible extensions of the MSSM in the context of type II string theory. We systematically investigate three-stack and four-stack quivers which realize the MSSM spectrum with the addition of a single MSSM singlet $S$ with an allowed $S H_{u} H_{d}$ term, which can lead to a dynamical electroweak-scale $\mu$-term. We present the three quivers which satisfy stringent string-theoretic and phenomenological constraints, including the presence of non-zero masses for all three families of quarks and leptons, the perturbative and non-perturbative absence of R-parity violating couplings and rapid dimension-five proton decay, and a mechanism for small neutrino masses. We find that these quivers can realize many models in the class of singlet-extended (supersymmetric) standard models, as D-instanton effects can in principle generate a superpotential of the form $f(S)$, where $f$ is a polynomial. Finally, we address the issue of the stabilization and decoupling of charged moduli which generically appear in D-instanton corrections to the superpotential.

KEYWORDS: Intersecting branes models, D-branes, Superstring Vacua 


\section{Contents}

1 Introduction 1

2 Quivers in type II orientifold compactifications 3

3 Beyond the MSSM 4

3.1 Singlet-extended standard models and string theory 5

$\begin{array}{lll}3.2 & \text { Model-independent singlet-extended quivers } & 7\end{array}$

$\begin{array}{lll}4 & \text { Conclusions } & 10\end{array}$

$\begin{array}{ll}\text { A The systematic analysis: methodology and constraints } & 11\end{array}$

$\begin{array}{ll}\text { B Stabilization and decoupling of charged moduli } & 14\end{array}$

\section{Introduction}

It has long been recognized that string theory naturally realizes important ingredients seen in particle physics, including gauge symmetry and chiral matter. More recently, the field has progressed tremendously and it is now possible to investigate stringy effects which could give rise to the finer details seen in particle physics. D-brane instantons [1-4] have been particularly fruitful in this regard, as they give non-perturbative superpotential corrections which could explain the large mass hierarchies [5-7] and mixing angles seen in the standard model, in addition to possibly playing a role in supersymmetry breaking $[8,9]$, and moduli stabilization [10-12]. Furthermore, they often generate operators relevant for obtaining small neutrino masses, including a Majorana mass term $([1,3,13-15])$, a small Dirac mass term [16], or a stringy Weinberg operator [17].

With this progress in our understanding of string vacua and the start-up of the LHC, it is important to consider the possible implications of string theory for particle physics in as much detail as possible. The natural starting place is to consider models which realize the exact MSSM, possibly extended by three right-handed neutrinos, and much work has been conducted along these lines. However, it is also important to consider the question of what allowed extensions of the standard model or MSSM are likely to occur in the string landscape. The minimal MSSM spectrum is not necessarily phenomenologically preferred, as the MSSM has some fine-tuning problems of its own, especially the $\mu$-problem. Furthermore, string constructions frequently contain extra chiral matter beyond the MSSM spectrum, which are often MSSM singlets. There is an extensive literature of phenomenological studies of such singlets, especially those which allow for a dynamical solution to the $\mu$ problem, ${ }^{1}$ but relatively little study from the string perspective. ${ }^{2}$

\footnotetext{
${ }^{1}$ See, e.g., [18-24]. For recent reviews, see [25-28].

${ }^{2}$ See $[29,30]$ for recent studies in heterotic constructions.
} 
Rather than considering full string models, we work from the bottom-up [31-33] at the level of quivers [5-7, 34-38], which allow for the efficient classification of many important physical effects. The data associated with a quiver essentially amounts to the gauge symmetry and matter content, which are data naturally associated with any semi-realistic string model. However, one can also examine the possible presence or absence of couplings and other physical effects by examining quantum numbers associated with the quiver, without having to delve into the geometric specifics of a fully defined string model. In this sense, we probe the "quiver landscape" for physical effects, hoping to identify promising quivers which could arise at various points in the landscape of string vacua.

Such an investigation was carried out for the exact MSSM and its extension by three right-handed neutrinos in $[17,36]$. Around fifty quivers were found with this matter spectrum which satisfy necessary constraints for the cancellation of non-abelian anomalies and a massless hypercharge, as well as allowing for Yukawa couplings for all three families of quarks and leptons via D-instanton effects. In those quivers, instantons whose presence is required to induce Yukawa couplings do not give rise to phenomenological drawbacks, such as violation of R-parity, rapid dimension-five proton decay, or a $\mu$-term which is too large. Additionally, each admits a mechanism which can give rise to neutrino masses of the correct order, and many contain semi-realistic mass hierarchies for the quarks and leptons.

In this work we perform an analysis of quivers whose spectrum is given by the exact MSSM extended by a singlet $S$, as well as quivers where this spectrum is further extended by the addition of three right-handed neutrinos. Models with such a spectrum belong to the class of so-called singlet-extended standard models. Such extensions are phenomenologically motivated as a solution to $\mu$-problem, where the $\mu$-term can be dynamically generated by a coupling $S H_{u} H_{d}$ when the $S$ field obtains a vacuum expectation value. In addition, singlet extensions are motivated from string theory by the simple fact that one or more standard model singlets often appear in the massless spectrum.

We present the three singlet-extended quivers with less than five stacks which satisfy a host of string theoretic and phenomenological constraints, including those mentioned above. We consider possible instanton corrections to the superpotential, which would determine the particular singlet-extended standard model that the quiver realizes, and find that the superpotential corrections are generically a polynomial function $f(S)$. Thus, the quivers themselves are model independent and could give rise to a variety of particular singlet-extended models when embedded in a global string model. The determination of the particular singlet-extended model associated with a global model depends heavily on the geometry of the Calabi-Yau, and we leave this analysis for future work.

We also address the issue of the stabilization of charged moduli which arise in instanton corrections to the superpotential. These moduli transform under the gauge symmetry of the D-brane which the D-instanton intersects. Assuming that the uncharged moduli are stabilized at high scale in some hidden sector where SUSY is broken, we examine the $F$-term and $D$-term contributions to the scalar potential in detail. Minimization of the potential shows that the real part of the charged moduli stabilizes near zero VEV, fixes the $D$-term near zero, and decouples from the low energy effective action.

This paper is organized as follows. In section 2, we review that basics of quivers and 
D-instantons in type II orientifold compactifications and discuss what can be said about non-perturbative corrections at the level of a quiver. In section 3, we discuss some basic motivations for singlet-extended standard models and discuss the role of instantons in the non-perturbative generation of crucial superpotential terms. We also present three quivers which satisfy many string theoretic and phenomenological constraints, discussing possible superpotential corrections that determine the type of singlet-extended standard model and neutrino masses. In appendix A, we explicitly present the constraints which the three quivers satisfy and discuss the methodology of our systematic analysis. Finally, in appendix B, we show that the real part of the charged moduli associated with instanton corrections generically stabilize near zero VEV and decouple from the low energy effective action.

\section{Quivers in type II orientifold compactifications}

The quivers analyzed and presented in this work fit naturally into orientifold compactifications of type II string theory [39-41]. ${ }^{3}$ For the sake of concreteness we consider type IIa, where gauge theories live on D6-branes which wrap four-dimensional Minkowski space and a three-cycle in the internal Calabi-Yau threefold. The D-branes carry Ramond-Ramond charge, which must be cancelled by the introducton of orientifold planes, whose locations are given by the fixed point loci of an antiholomorphic involution on the internal space. The cancellation of Ramond-Ramond charge, also known as tadpole cancellation, is a condition on the homology of the D6-branes and O6-planes.

D6-branes naturally give rise to gauge symmetry and chiral matter. When wrapped on a generic cycle, a stack of $N$ D6-branes gives rise to $\mathrm{U}(N)=\mathrm{SU}(N) \times \mathrm{U}(1)$ gauge symmetry. Additionally, $N$ D6-branes on cycles which are homologically-fixed or pointwise-fixed by the orientifold action give rise to $\mathrm{Sp}(2 N)$ and $\mathrm{SO}(2 N)$ gauge symmetry, respectively. Chiral matter arises in the bifundamental representation at the non-trivial intersection of two generic D6-branes, and it is also possible to have symmetric or anti-symmetric tensor representations where a D6-brane intersects its image brane under the orientifold action. Furthermore, two given D6-branes might intersect in multiple points on the compact internal space, giving rise to multiple families, where the number of families is the topological intersection number of the two D6-branes in the Calabi-Yau.

Of great phenomenological importance is the generalized Green-Schwarz mechanism, which generically gives a Stückelberg mass to the gauge bosons associated with the U(1) symmetries of the D6-branes, but might leave some linear combination massless, depending on the homology of the D-branes and O-planes. The presence of such a massless U(1) is crucial in type II model building, as it can be interpreted as hypercharge. Additionally, the $\mathrm{U}(1)$ combinations which do receive a mass still exist as global symmetries of the theory, which charge chiral matter and generically forbid many Yukawa couplings at the perturbative level in the superpotential. In the absence of non-perturbative corrections, this would yield some number of massless quark and lepton families.

However, in [1] it was shown that Euclidean D2-instantons, which are pointlike in spacetime and wrap a three-cycle in the Calabi-Yau, can non-perturbatively generate a

\footnotetext{
${ }^{3}$ For examples of globally consistent supersymmetric standard-like models, see [42, 43].
} 
superpotential coupling of the generic form

$$
W \sim e^{-S_{E 2}^{c l}} \prod_{i} \Phi_{i}
$$

where the $\Phi_{i}$ are matter fields charged under the gauge group of some D6-branes. In addition to generating couplings which might be perturbatively forbidden, it has been shown that such E2-instantons can generate phenomenologically relevant couplings which are always perturbatively forbidden in type II, such as the $10105_{H}$ top-quark Yukawa coupling in SU(5) GUT models [44] or a Majorana mass term for right-handed neutrinos $([1,3,13-15])$. Furthermore, D-instantons can also account for the observed order of the neutrino masses by inducing [17] a Weinberg operator [45] directly (for a lower string scale) or via a highly suppressed Dirac mass term [16].

Determining the form of instanton-induced superpotential corrections, if any, requires a careful analysis of the uncharged and charged fermionic zero modes, respectively in the E2-E2 and E2-D6 open string sectors. In addition to the $x^{\mu}$ and $\theta^{\alpha}$ modes which are present in the measure of the superpotential, the uncharged modes include deformation modes and a $\bar{\tau}_{\dot{\alpha}}$ mode which must be projected out or lifted in order for the instanton to generate a superpotential contribution. This occurs, for example, when the instanton wraps a rigid orientifold invariant cycle, which is a condition that depends heavily on geometric specifics. Thus, it is not possible to address these zero modes merely from a quiver analysis.

The charged zero modes, on the other hand, are charged under the gauge groups of the D6-branes and thus have quantum numbers which can be seen at the quiver level. For example, consider the simple case of three $\mathrm{U}(1)$ stacks of D6-branes $a, b$, and $c$, with fields $\Phi$ and $\Psi$ which transform as $(a, \bar{b})$ and $(b, \bar{c})$. In that case the coupling

$$
\Phi_{(1,-1,0)} \Psi_{(0,1,-1)}
$$

has non-zero charge under the global U(1)'s, which are denoted by subscripts, and is therefore perturbatively forbidden. Any instanton which might generate the coupling nonperturbatively must cancel the $\mathrm{U}(1)$ charge, and thus must give rise to a $\bar{\lambda}_{a}$ and a $\lambda_{c}$ charged mode. In this way, it is straightforward to determine not only which charged modes must be present if an instanton is to generate a particular superpotential coupling, but also which superpotential couplings might be generated by the same instanton. These necessary conditions can all be seen at the quiver level, without having to specify the geometry of a particular Calabi-Yau.

\section{Beyond the MSSM}

Since the quiver approach allows for the investigation of many physical effects without the necessity of specifying a particular string geometry, it is worthwhile to examine quivers which realize promising phenomenological models. The natural starting point is with quivers which realize the exact spectrum of the MSSM, possibly extended by three right-handed neutrinos. A systematic investigation of MSSM quivers at the level of gauge symmetry and matter content was performed in [46], and a systematic analysis of realistic MSSM quivers 
was first performed at the level of couplings in $[17,36]$, taking into account non-perturbative D-instanton effects and extensive phenomenological considerations.

While those MSSM quivers are phenomenologically promising and provide an excellent starting place for string model-building, there are motivations for adding additional chiral matter to the spectrum. For example, the MSSM superpotential contains a term $\mu H_{u} H_{d}$. The $\mu$ parameter must be non-zero and above $\sim 100 \mathrm{GeV}$ to ensure sufficiently large Higgsino masses, while the associated soft term must be non-zero to ensure that both $H_{u}$ and $H_{d}$ obtain nonzero vacuum expectation values after electroweak symmetry breaking. Both should be below the TeV scale to allow a solution to the Higgs hierarchy problem. However, the natural cutoff scale is the Planck scale or some other high scale, and the associated fine-tuning problem is known as the $\mu$ problem [47]. In addition to possibly solving the $\mu$ or other phenomenological problems, the addition of extra matter to the spectrum and/or additional gauge symmetries are certainly allowed within the context of string theory and often occur in concrete constructions.

\subsection{Singlet-extended standard models and string theory}

A well-motivated class of extensions to the standard model are broadly known as singletextended standard models. We are concerned here with the supersymmetric versions, which extend the chiral spectrum of the MSSM by some number of MSSM singlets $S_{i}$. We consider the two specific cases where the exact MSSM spectrum and the MSSM spectrum extended by three right-handed neutrinos are augmented by the addition of a single $S$ field. Phenomenologically, one reason for adding such a field is to generate the $\mu$-term dynamically via the superpotential term $\lambda S H_{u} H_{d}$, where the $\mu$-term will be $\mu \sim \lambda\langle S\rangle$ upon $S$ getting a VEV. Since $S, H_{u}$, and $H_{d}$ are coupled in the scalar potential, the VEV of $S$ is typically of electroweak scale up to a few TeV, providing an acceptable $\mu$ if $\lambda$ is not too small. We therefore expect $\lambda \sim O(1) .{ }^{4}$

Particular phenomenological models within the class of singlet-extended standard models are broadly classified by the form of superpotential terms involving $S$, which play a crucial role in phenomenology. We write the superpotential as

$$
W=Y_{u} q_{L} H_{u} u_{R}+Y_{d} q_{L} H_{d} d_{R}+Y_{l} L H_{d} E_{R}+\lambda S H_{u} H_{d}+W_{\nu}+W_{\text {model }},
$$

which contains the quark and lepton Yukawa couplings of the MSSM superpotential, as well as the term which generates the $\mu$-term dynamically, some terms $W_{\nu}$ which account for the neutrino masses, and some terms $W_{\text {model }}$ which are dependent upon the particular singlet-extended model. The simplest include the nearly minimal supersymmetric standard model (nMSSM [21-24]), the $S^{2}$ model, and the next-to-minimal supersymmetric standard

\footnotetext{
${ }^{4}$ Other natural solutions to the $\mu$ problem include the Giudice-Masiero mechanism [48] and the direct generation of $\mu$ by string instantons [1].
} 
model $^{5}$ (NMSSM [18]), which have superpotential contributions of the form ${ }^{6}$

$$
\begin{aligned}
\operatorname{nMSSM}: & W_{\text {model }}=c_{s} S \\
S^{2}: & W_{\text {model }}=\alpha S^{2} \\
\mathrm{NMSSM}: & W_{\text {model }}=\kappa S^{3} .
\end{aligned}
$$

Another possibility, not considered in this paper, is the UMSSM, which involves an extra non-anomalous $\mathrm{U}(1)^{\prime}$ gauge symmetry $[19,20,49]$. Each of these models have interesting phenomenological implications. For example, the extended Higgs sector associated with $S$ may significantly modify the existing and future searches for the Higgs because of decays into pairs of light pseudoscalars [50] or because of reduced couplings due to mixing with the singlet [51]. Similarly, the larger neutralino sector may lead to extended cascades at the LHC or modified cold dark matter scenarios, while the soft cubic supersymmetry breaking term associated with $\lambda S H_{u} H_{d}$ greatly facilitates the possibility of the strong first-order phase transition needed for electroweak baryogenesis [25-28].

While it is clear that string theory can realize singlet-extended standard models at the level of the spectrum, since singlets so often appear in string constructions, it is interesting to ask more model-dependent questions related to the presence of certain superpotential terms. If a singlet-extended standard model is to arise in type II string theory, the singlet $S$ carries global U(1) charge under the branes and thus a coupling of the form $S^{n}$ is perturbatively forbidden. Fortunately, a D-instanton which generates these terms nonperturbatively gives corrections of the form

$$
e^{-S_{E 2}^{c l}} M_{s}^{3-n} S^{n} \equiv c_{n} S^{n}
$$

which allow for many types of singlet-extended models, including the ones described above. Moreover, it is worth noting that though a single instanton does not have the correct charged zero mode structure to generate multiple $S^{n}$ terms with differing $n$, this can be achieved by superpotential corrections from multiple instantons with different intersection numbers with the gauge branes.

The presence of a term of the form $S^{n}$ ensures the absence of a massless pseudoscalar associated with the Peccei-Quinn symmetry which the term explicitly breaks. In these models the prefactor $c_{n}$ must be sufficiently large for $n=1,2,3$ to ensure that the mass of the pseudoscalar is sufficiently large. One expects the mass to be ${ }^{7}$

$$
M_{A}^{2} \sim c_{n}\langle S\rangle^{n-1},
$$

\footnotetext{
${ }^{5}$ The problem of cosmological domain walls due to the spontaneously broken $\mathbb{Z}_{3}$ symmetry in the original version of the NMSSM [27, 28] may be resolved here, e.g., by the presence of other instantoninduced operators.

${ }^{6}$ The coefficients for the models in (3.2) should be nonzero to avoid an unwanted global symmetry and associated massless pseudoscalar. The latter is "eaten" in the UMSSM. The quartic terms in the NMSSM for $\kappa \neq 0$ (or the $D$ term in the UMSSM) prevent a runaway potential in the $S \rightarrow \infty, H_{u, d}=0$ direction. In the other cases the absence of the runaway direction places constraints on the soft mass-squared term for $S$.

${ }^{7}$ We have assumed that the coefficients of the of the soft supersymmetry breaking terms corresponding to $c_{n} S^{n}$ are $c_{n} A_{n}$ where $A_{n} \sim\langle S\rangle$. For a detailed treatment of the $n=1,3$ cases, see [51].
} 
and a reasonable range for the mass is $M_{A} \sim(3 \mathrm{GeV}-1 \mathrm{TeV})$. Ensuring that the mass is in this range for $\langle S\rangle=1 \mathrm{TeV}$ requires $c_{1} \sim(3 \mathrm{GeV}-1 \mathrm{TeV})^{2}, c_{2} \sim(0.01 \mathrm{GeV}-1 \mathrm{TeV})$ and $c_{3} \sim\left(10^{-5}-1\right)$. In a particular quiver, an important question is whether or not the instanton suppression factors allow for $c_{n}$ 's in these ranges. We will answer this explicitly in our examples.

\subsection{Model-independent singlet-extended quivers}

Relative to the breadth of possibile particle physics models afforded by string theory, these three singlet-extended models have far more in common than they do differences, and it is interesting to study with what frequency and under what conditions these models might arise in the string landscape. We now investigate this with a detailed quiver analysis at the level of couplings and will present three singlet-extended quivers, each of which can accomodate the nMSSM, the $S^{2}$ model, the NMSSM, or a more general theory with $W_{\text {model }}$ a polynomial in $S$.

We performed two systematic analyses of three-stack and four-stack quivers, one which realizes the exact MSSM chiral spectrum, and one which realizes the exact MSSM extended by three right-handed neutrinos. Both required stringent string theoretic and phenomenological constraints on the quivers, and all surviving quivers have non-zero masses for all three families of quarks and leptons, as well as no R-parity violation or rapid dimension-five proton decay at either the perturbative or non-perturbative level. Additionally, the quivers have natural mechanisms for realizing the correct order of the top-quark Yukawa coupling and the neutrino masses. For more details on the methodology and constraints, we refer the reader to appendix A.

Interestingly, there are only three ${ }^{8}$ three-stack or four-stack quivers, given in table 1 , which satisfy all of the string theoretic and phenomenological constraints. All have the exact MSSM spectrum extended by three right-handed neutrinos and a singlet $S .{ }^{9}$ Furthermore, all exhibit the well-known Madrid embedding [52] of the hypercharge,

$$
\mathrm{U}(1)_{Y}=\frac{1}{6} \mathrm{U}(1)_{a}+\frac{1}{2} \mathrm{U}(1)_{c}+\frac{1}{2} \mathrm{U}(1)_{d},
$$

where there are four stacks of D-branes $a, b, c$, and $d$ with $\mathrm{U}(3)_{a} \times \mathrm{U}(2)_{b} \times \mathrm{U}(1)_{c} \times$ $\mathrm{U}(1)_{d}$ gauge symmetry, which becomes $\mathrm{SU}(3)_{c} \times \mathrm{SU}(2)_{L} \times \mathrm{U}(1)_{Y}$ due to the GreenSchwarz mechanism.

In particular, this means that there are no quivers with the exact MSSM spectrum plus a singlet $S$ which satisfy all of the constraints. Two of the quivers with that spectrum nearly pass all constraints, but in those cases the $\mu$-term is directly generated by an instanton whose presence is necessary to generate an up-flavor quark Yukawa coupling. As the

\footnotetext{
${ }^{8}$ The scarcity of singlet-extended quivers relative to the $\sim 50$ MSSM quivers of previous work is due to additional constraints and the investigation here of only three-stack and four-stack quivers. It is not that the MSSM is more common in string constructions.

${ }^{9}$ We require that the right-handed neutrinos and the singlet $S$ can be distinguished from one another. This is accomplished by having them transform differently under the D-brane gauge groups and by ensuring the absence of a Dirac-type mass coupling $L H_{u} S$. See appendix A for more details.
} 


\begin{tabular}{|c|c|c|c|c|c|c|c|c|c|c|c|c|c|c|}
\hline \multirow{2}{*}{ Quiver \# } & \multicolumn{2}{|c|}{$q_{L}$} & \multirow{2}{*}{$\begin{array}{c}d_{R} \\
(\bar{a}, c)\end{array}$} & \multicolumn{2}{|c|}{$\overline{u_{R}}$} & \multirow{2}{*}{$\begin{array}{c}L \\
(b, \bar{d})\end{array}$} & \multicolumn{3}{|c|}{$E_{R}$} & \multicolumn{2}{|c|}{$N_{R}$} & \multirow{2}{*}{$\begin{array}{c}H_{u} \\
(b, d)\end{array}$} & \multirow{2}{*}{$\begin{array}{c}H_{d} \\
(\bar{b}, \bar{c})\end{array}$} & \multirow{2}{*}{$\begin{array}{c}S \\
(c, \bar{d})\end{array}$} \\
\hline & $(a, b)$ & $(a, \bar{b})$ & & $(\bar{a}, \bar{c})$ & $(\bar{a}, \bar{d})$ & & $(c, d)$ & $\square \square_{c}$ & $\square \square_{d}$ & $\boxminus_{b}$ & $\bar{\theta}_{b}$ & & & \\
\hline 1 & 2 & 1 & 3 & 2 & 1 & 3 & 2 & 1 & 0 & 3 & 0 & 1 & 1 & 1 \\
\hline 2 & 2 & 1 & 3 & 2 & 1 & 3 & 0 & 2 & 1 & 3 & 0 & 1 & 1 & 1 \\
\hline 3 & 0 & 3 & 3 & 0 & 3 & 3 & 1 & 0 & 2 & 0 & 3 & 1 & 1 & 1 \\
\hline
\end{tabular}

Table 1. The three surviving quivers. Each quiver has its matter content and transformation behavior specified by a single row, and all have the exact MSSM spectrum extended by three right-handed neutrinos and a singlet $S$.

dynamic generation of the $\mu$-term is a main goal in examining singlet-extended standard models, we do not consider those quivers.

Since the three quivers which do survive avoid many serious phenomenological pitfalls, it is worth investigating which particular singlet-extended standard models are possible for these quivers by investigating which of the terms $S^{n}$ might be generated by nonperturbative D-instanton effects. A quick look at the quivers gives the global U(1) charge of such couplings to be

$$
Q_{a}\left(S^{n}\right)=0 \quad Q_{b}\left(S^{n}\right)=0 \quad Q_{c}\left(S^{n}\right)=n \quad Q_{d}\left(S^{n}\right)=-n,
$$

so that an instanton $E 2_{n}$ which intersects the four gauge D-branes as

$$
I_{E 2_{n}, a}=0 \quad I_{E 2_{n}, b}=0 \quad I_{E 2_{n}, c}=n \quad I_{E 2_{n}, d}=-n
$$

will generate an $S^{n}$ coupling of the form (3.3), with a different suppression factor associated with each instanton. Though this ensures that it is possible to generate the superpotential terms associated with the nMSSM, the $S^{2}$ model, or the NMSSM, it does not ensure that the instanton suppression factor, which depends on the volume of the three-cycle which the instanton wraps, is such that they are of an allowed order.

In fact, one might question whether it is even possible at the level of quivers to address this issue. Certainly it is not possible to say what the volume of the instanton cycle is from geometric specifics and stabilization arguments, since we have not specified any geometry, but it turns out that the issue can be addressed phenomenologically. For example, it was pointed out in [34] that in some MSSM quivers, the instanton which generates the $\mu$-term is also required to generate a quark or lepton Yukawa coupling. To generate a Yukawa coupling of the correct order would require the suppression factor $e^{-S_{E 2}^{c l}} \gtrsim 10^{-5}$, which is not nearly suppressed enough to generate a $\mu$-term $e^{-S_{E 2}^{c l}} M_{s} H_{u} H_{d}$ of the correct order, and the quiver must be phenomenologically ruled out. ${ }^{10}$

A similar analysis of the charged zero mode structure of instantons which generate an $S^{n}$ coupling shows that only one of these instantons might also generate a quark or lepton Yukawa coupling. Specifically, the instanton $E 2_{1}$ which generates a superpotential term linear in $S$ will generate a charged lepton Yukawa coupling $L H_{d} E_{R}$ in either of the first two

\footnotetext{
${ }^{10}$ Such a quiver would be possible for a lower string scale, $M_{s} \lesssim 10^{7} \mathrm{GeV}$. See [17] for an analysis of related issues in type II orientifold compactifications.
} 
quivers in table 1. For phenomenological reasons the $c_{s}$ parameter of the nMSSM must be $O\left(\mathrm{TeV}^{2}\right)$, though, which requires a suppression factor $e^{-S_{E 2}^{c l}} \sim 10^{-30}$, and thus $E 2_{1}$ cannot simultaneously account for the correct order of the charged lepton Yukawa coupling and $c_{s}$. However, this same lepton coupling might also receive a contribution from an instanton with an extra pair of charged vector-like zero modes $\lambda_{c}$ and $\bar{\lambda}_{c}{ }^{11}$ If such an instanton with vector-like zero modes accounts for the correct order of the lepton Yukawa coupling, then the suppression factor associated with $E 2_{1}$ is not fixed, and $E 2_{1}$ could generate a superpotential term linear in $S$ with any suppression factor. Furthermore, based on global $\mathrm{U}(1)$ charges, one might be concerned that the instanton which generates an $S^{n}$ coupling also generates an R-parity violating of the form $\left(q_{L} L d_{R}\right)^{n}$. Such a term will generically be suppressed by $a_{n} / M_{s}^{2 n}$, where the desirable superpotential coupling takes the form $a_{n} S^{n}$, and thus is not dangerous.

These three singlet-extended quivers are model independent, as any superpotential term $S^{n}$ can be generated non-perturbatively by a D-instanton, and can thus realize the nMSSM, the $S^{2}$ model, the NMSSM, or any other theory with $W_{\text {model }}=f(S)$, with $f$ a polynomial. Moreover, the corresponding instanton suppression factors are not phenomenologically fixed. Thus, the quivers serve as an excellent starting point for building global type II orientifolds with the spectrum of a singlet-extended standard model. Determination of which particular model is embodied by such a global type II orientifold would then be possible, as one could explicitly determine the form of instanton corrections to the superpotential via geometric and CFT techniques.

In addition to allowing one to determine the explicit superpotential corrections due to instantons, such a global construction also allows one to address in detail the values of the instanton suppression factors. This is accomplished by examining the details of Kähler moduli stabilization, where the vacuum expectation value of the real part of an appropriate Kähler modulus is the volume of the cycle which the instanton wraps, which is the crucial parameter that determines the instanton suppression factor. While it is not possible at the quiver level to state the precise value of the instanton suppression factors, since a global construction has not been specified and the Kähler moduli have not been stabilized, the ranges for $c_{1,2,3}$ discussed in section 3.1 which allow for a light pseudoscalar are certainly achievable due to the exponential supression. Alternatively, if the cycle which the instanton wraps is stabilized at a relatively small volume, $c_{n}$ could be large and allow for a high mass pseudoscalar. This is in contrast to the heterotic scenario studied in [30], where the vacuum expectation values of standard model singlets which determine $c_{3}$ typically give $c_{3} \ll 1$, and thus give a light pseudoscalar.

Finally, since these quivers exhibit many other nice phenomenological features, ${ }^{12}$ it is worth commenting on the possible forms which $W_{\nu}$ might take for each quiver in order to account for the observed order of the neutrino masses. In the first two quivers the Dirac neutrino mass term $L H_{u} N_{R}$ can be generated by two instantons, which we call $E 2$

\footnotetext{
${ }^{11}$ See [6] for more details on instantons with vector-like zero modes in quiver analyses.

${ }^{12}$ Beyond the requirement that the top mass be very massive relative to other MSSM matter fields, we have not addressed mass hierarchies or mixing angles in this analysis. The first two quivers, in particular, would require some amount of fine-tuning of worldsheet instantons to obtain realistic mixing angles.
} 
and $E 2_{v}$, where the latter has an additional pair of vector-like charged zero modes $\lambda_{d}$ and $\bar{\lambda}_{d}$. The observed order of the neutrino masses can be accounted for if $E 2_{v}$ generates a highly suppressed Dirac mass term. However, this is not true of E2, since its presence will also generically generate a Majorana mass term $N_{R} N_{R}$, giving rise to the seesaw mechanism. With $M_{s} \approx 10^{18} \mathrm{GeV}$, the associated seesaw mass would give a contribution to the neutrino mass a few orders of magnitude below what is observed, and thus for the seesaw mechanism to work at high string scale, $E 2_{v}$ must also generate a contribution to the Dirac mass term. ${ }^{13}$ In the third quiver, the Dirac mass is perturbatively allowed, and therefore the option of a highly suppressed non-perturbative Dirac mass is not available. The seesaw mechanism can be realized, however, if a Majorana mass term is generated by an instanton.

\section{Conclusions}

String theory naturally gives rise to two of the most important features of experimental particle physics, namely gauge symmetry and chiral matter. Recently, however, improved knowledge of non-perturbative effects which are present in string models has been used to explain finer details of particle physics, such as hierarchical Yukawa couplings. This progress has led to a great deal of work in bottom-up string model building in the context of type II orientifold compactifications. However, it is also important to study the MSSM and its most likely extensions from the perspective of the string landscape.

Of great use has been the notion of a quiver, which is a subset of the data associated with a string model that allows for the efficient investigation of physical effects at the level of couplings. Systematic phenomenological studies of MSSM quivers have been performed which investigate the role of D-instantons in generating quark and lepton Yukawa couplings while still avoiding phenomenological pitfalls, such as R-parity violating couplings and rapid dimension-five proton decay.

In this work, we moved beyond the MSSM and investigated the class of singlet-extended (supersymmetric) standard models, which add a singlet $S$ to the MSSM spectrum, possibly also extended by three right-handed neutrinos. Phenomenologically, the motivations include the dynamical generation of the $\mu$-term by a coupling $S H_{u} H_{d}$ when $S$ gets a vacuum expectation value, as well as interesting possibilities for extended Higgs and neutralino sectors, with implications for the LHC, cold dark matter, and electroweak baryogenesis. Such extensions are also motivated by string models, which often contain one or many MSSM singlets. The nMSSM, $S^{2}$ model, and NMSSM are notable examples of singletextended standard models, with the difference between the three being the structure of superpotential terms involving the $S$ field.

It was the goal of this paper to efficiently map out regions of the landscape which might allow for singlet-extended models, taking into account non-perturbative effects. In section 2, we reviewed the basics of quivers in type II orientifold compactifications as well as

\footnotetext{
${ }^{13}$ This issue of the same instanton generating the Dirac mass term and the Majorana term was discussed in depth in [17], where it was shown that the string scale must be lowered for the corresponding seesaw mass to generate neutrino masses of the observed order.
} 
non-perturbative superpotential corrections due to D-instantons. In section 3 we motivated the class of singlet-extended standard models and discussed the role of instanton effects in generating superpotential terms of the form $S^{n}$.

We then presented the three four-stack singlet-extended quivers which satisfy all of the string theoretic and phenomenological constraints in appendix A, which include the presence of non-zero masses for all three families of quarks and leptons and of a perturbative $S H_{u} H_{d}$ term, and the absence of R-parity violating couplings and rapid dimension-five proton decay at both the perturbative and non-perturbative level. All three of these quivers exhibit the spectrum of the MSSM extended by three right-handed neutrinos and a singlet $S$ and furthermore have the Madrid hypercharge embedding. Moreover, all three quivers generically allow for the non-perturbative generation of a superpotential of the form $f(S)$, where $f$ is a polynomial. In this case, each monomial would be generated by a different instanton with a suppression factor that is not phenomenologically fixed, and thus the quivers are completely model independent at the level of the superpotential. Thus, they can in principle realize the nMSSM, the $S^{2}$ model, and the NMSSM. Furthermore, all have mechanisms for small neutrino masses.

This work has shown the efficiency of the quiver approach in identifying promising patches of the string landscape. It would be interesting to construct a global type II orientifold compactification which realizes one of these quivers, which would allow for the classification of instanton corrections to the superpotential in that particular string model and thus the explicit determination of $f(S)$ in that model. It would also be interesting to conduct similar studies of the quiver landscape using other promising phenomenological models, such as singlet-extended models involving an additional non-anomalous $\mathrm{U}(1)^{\prime}$ gauge symmetry $[19,20,49]$.

\section{Acknowledgments}

We acknowledge useful conversations with Robert Richter and are grateful for his participation in past collaborations. We acknowledge the hospitality of the KITP during the Strings at the LHC and in the Early Universe program for providing a stimulating environment during the completion of this work. This research was supported in part by the National Science Foundation under Grant No. PHY05-51164. The work of M.C. and J.H. is supported by the DOE Grant DOE-EY-76-02-3071, the NSF RTG grant DMS-0636606, the Fay R. and Eugene L. Langberg Chair and in part by the Slovenian Research Agency (ARRS). The work of P.L. is supported by the IBM Einstein Fellowship and by the NSF grant PHY-0503584.

\section{A The systematic analysis: methodology and constraints}

Having motivated the use of a quiver analysis in identifying promising vacua in the string landscape, in this appendix we briefly describe the methodology and constraints employed in the systematic search which produced the quivers presented in the main text. While the quivers are not specific to type II string theory at the level of gauge symmetry and chiral 
matter, the phenomenological analysis strongly relies on the presence of global U(1) symmetries, possible non-perturbative superpotential corrections due to D-instanton effects, and a $\mathrm{U}(1)_{Y}$ gauge symmetry which is not given a mass by the generalized Green-Schwarz mechanism. These ideas naturally arise in type II, motivating the use of its language rather than the language of nodes and arrows. The map is straightforward, though, as a stack of D-branes give rise to a node, and an open string state between two stacks of intersecting D-branes gives rise to an arrow between two nodes.

Methodology. A systematic phenomenological analysis of the type II quiver landscape begins with a reasonably small set of input data. First, one must decide which phenomenological theory is of interest and choose the number of stacks with which to realize the gauge symmetry. For example, in the MSSM one needs a stack of three and a stack of two Dbranes ${ }^{14}$ to give the requisite $\mathrm{SU}(3)_{c}$ and $\mathrm{SU}(2)_{L}$, but one might also add many stacks which contain a single D-brane. We chose to examine the cases of one or two additional stacks with a single D-brane, giving a total of three stacks and four stacks.

In addition to the specification of the number and types of D-brane stacks, which gives the gauge symmetry of the theory, one must also specify the chiral matter spectrum. In the work of $[6,7,17,36]$, the authors considered the exact MSSM or the exact MSSM extended by three right-handed neutrinos. In this work we considered these two sets of spectra extended by an MSSM singlet $S$ which should not be interpreted as a righthanded neutrino.

As the MSSM and its extensions have a hypercharge $\mathrm{U}(1)_{Y}$ gauge symmetry and the $\mathrm{U}(1)$ gauge symmetries associated with a stack of D-branes are generically lifted by the generalized Green-Schwarz mechanism, it is also necessary to specify the linear combination $\mathrm{U}(1)_{Y}=\sum q_{x} \mathrm{U}(1)_{x}$ which is left massless. It turns out that the chiral matter spectrum along with necessary constraints for tadpole cancellation and a massless hypercharge put strong restrictions on the allowed linear combinations, often giving a finite number of combinations which can realize the hypercharge. This was the case in the analyses performed in this paper.

Given the number and type of D-brane stacks, the chiral matter spectrum of interest, and the massless linear combination of U(1)'s which is interpreted as hypercharge, it is possible to write down every way in which a given matter field might transform. For example, in the Madrid embedding, in (3.5), the right-handed down-quarks $d_{R}$ might be realized as $\bigoplus_{a},(\bar{a}, c)$, or $(\bar{a}, d)$. Given that there are three families and the fact that different families might arise in different representations of the D-brane gauge groups, it is possible to enumerate all possible ways in which three $d_{R}$ 's might transform. One could do this for every field in the chiral spectrum, which allows for the straightforward enumeration of all possible quivers with this number of stacks, matter spectrum, and hypercharge embedding.

Most of these quivers do not satisfy necessary string theoretic constraints for tadpole cancellation and a massless hypercharge. Additionally, quivers which do satisfy those constraints often exhibit undesirable phenomenological effects. For these reasons, we en-

\footnotetext{
${ }^{14}$ Unless the $\mathrm{SU}(2)_{L}$ factor of the MSSM is realized as an $\mathrm{Sp}(2) \cong \mathrm{SU}(2)$ gauge symmetry by a single D-brane on a three-cycle homologically invariant under the orientifold action.
} 
force extensive constraints which ensures that the surviving quivers are theoretically and phenomenologically viable.

Theoretical and phenomenological constraints. In this work we performed two systematic analyses, with the difference being the presence or absence of right-handed neutrinos in the spectrum. This difference motivates slightly different phenomenological constraints, so we first discuss the constraints common to both analyses and then discuss the additional constraints placed on the analysis of quivers which exhibit right-handed neutrinos. We briefly discuss constraints which were used in previous analyses, referring the reader to $[6,7,17,36]$ for more details, and discuss new constraints related to the presence of a singlet $S$ in the spectrum.

As mentioned in section 2, the cancellation of Ramond-Ramond charge in the CalabiYau, also known as tadpole cancellation, places constraints on the homology of the D-branes and O-planes. There are also additional constraints on the homology of the D-branes and O-planes if a linear combination of U(1)'s is to be left massless by the Green-Schwarz mechanism. Since the homology of D-branes determines the chiral matter spectrum of the theory, these two constraints each place a constraint on the chiral matter spectrum which must be satisfied if the quiver is to cancel tadpoles and have a massless hypercharge when embedded in a top-down string model. In previous work, these have been called "topdown" constraints not because top-down globally consistent string models were presented, but because the constraints on the chiral matter arise from string theory. The constraints on the chiral matter which arise from string theory are equivalent to non-abelian and abelian anomaly cancellation in the low energy effective theory, so they can also be viewed as bottom-up.

In addition, we require that the quivers satisfy many phenomenological constraints. First, since many quark and lepton Yukawa couplings are perturbatively forbidden, we require that enough of these forbidden couplings are non-perturbatively generated by Dinstantons to ensure non-zero masses for all three families of quarks and leptons. However, these same instantons which are required to generate Yukawa couplings might also generate phenomenological drawbacks. Therefore, we require the absence of the R-parity violating couplings $d_{R} d_{R} u_{R}, L L E_{R}, q_{L} L d_{R}$, and $L H_{u}$ and the absence of the dimension-five proton decay operators $q_{L} q_{L} q_{L} L$ and $u_{R} u_{R} d_{R} E_{R}$ on both the perturbative and non-perturbative level. We also require that there is a natural explanation for the size of the top-quark Yukawa coupling. These constraints were also present in systematic analyses presented in previous work.

We further require that three new constraints are satisfied which maintain the motivations for looking at singlet-extended standard models. First, since we wish to have a dynamical $\mu$-term, we require that the coupling $H_{u} H_{d}$ is absent at both the perturbative and non-perturbative levels, while $S H_{u} H_{d}$ is perturbatively realized. In addition, we require that the Dirac-type coupling $L H_{u} S$ is absent at both levels, so that the singlet $S$ should not be interpreted as a right-handed neutrino. This allows us to isolate the issues of neutrino mass and the scale of the $\mu$-term. Finally, we require that either a linear, quadratic, or cubic term in $S$ can be generated in the superpotential without giving rise to the phenomenological drawbacks mentioned in the previous paragraph. 
In addition, for the analysis which considers quivers with three right-handed neutrinos, we require that a linear term in $N_{R}$ is not generated by an instanton whose presence is required to generate a forbidden Yukawa coupling. We also require that $S$ and $N_{R}$ are realized in different D-brane sectors and thus do not transform in the same way, ensuring the $S$ is distinguishable from $N_{R}$.

\section{B Stabilization and decoupling of charged moduli}

In this appendix we address the issue of the stabilization and decoupling of charged moduli $C_{i}$. Given the specific assumption that the stabilization of uncharged moduli $U_{i}$ (and supersymmetry breaking) takes place at a scale $\gg \mathrm{O}(\mathrm{TeV})$, we deduce that the real part of charged moduli is subsequently determined by the D-flatness conditions while the imaginary part of charged moduli is fixed at the $\mathrm{TeV}$ scale due to the D-instanton induced couplings to the charged matter $\Phi_{i}$. For simplicity, we shall denote the collection of charged moduli, uncharged moduli and matter fields with the single set of letters $C, U$ and $\Phi$, respectively.

In the type II context charged moduli arise in non-perturbative corrections to the superpotential from D-instantons which have chiral intersections with one or more gauge D-branes. For example, recall that in type IIa the superpotential correction generated by an instanton wrapping a three-cycle $\Xi$ is suppressed by [1]

$$
e^{-S_{E 2}^{c l}}=\exp \left[\frac{2 \pi}{l_{s}^{3}}\left(\frac{1}{g_{s}} \operatorname{Vol}_{\Xi}-i \int_{\Xi} C_{3}\right)\right] .
$$

If $\Xi$ intersects a D6-brane wrapping $\Pi_{a}$, the Ramond-Ramond three-form $C_{3}$ participates in the Chern-Simons couplings which cancel anomalies associated with $\mathrm{U}(1)_{a}$ via the generalized Green-Schwarz mechanism. The corresponding transformation behavior of the three-form gives the transformation behavior

$$
e^{-i \int_{\Xi} C_{3}} \mapsto e^{i Q_{a}(E 2) \Lambda_{a}} e^{-i \int_{\Xi} C_{3}},
$$

where $\Lambda_{a}$ parameterizes the transformation of the $\mathrm{U}(1)_{a}$ gauge boson $A_{\mu}$ via $A_{\mu} \mapsto A_{\mu}+$ $\partial_{\mu} \Lambda_{a}$, and $Q_{a}(E 2)=N_{a} \Xi \cdot\left(\Pi_{a}-\Pi_{a}^{\prime}\right)$. In this sense, we refer to the corresponding modulus as a charged modulus. For explicit moduli dependence of three-cycle volumes in the toroidal type IIa context see, e.g., [53].

For the sake of simplicity, here we parametrize the instanton suppression factor associated with a non-perturbative superpotential correction as $e^{-a U-b C}$, where $U$ parameterizes uncharged complex structure moduli and $C$ is a specific charged modulus associated with the $\mathrm{U}(1)_{a}$ factor of gauge branes $D_{a}$ wrapping a specific three-cycle $\Pi_{a}$. In the type IIa context the uncharged part of the non-perturbative coupling parameterizes the complex structure and dilaton moduli associated with those three-cycles which do not intersect with the specific three-cycle $\Pi_{a}$.

For specificity we assume that the uncharged moduli are stabilized at a scale $\gg \mathrm{TeV}$, with the superpotential and Kähler potential split as

$$
W=W_{0}(\Phi, U, C)+\tilde{W}(U) \quad K=K_{0}\left(U, U^{*}, C+C^{*}+Q_{a} V, \Phi, \Phi^{*}\right)+\tilde{K}\left(U, U^{*}\right),
$$


where $\Phi$ is a chiral field charged under the gauge symmetry of the D-brane with anomalous $\mathrm{U}(1)_{a}$. The vector super-multiplet $V$, associated with $\mathrm{U}(1)_{a}$, gets a Stückelberg mass of the order $\tilde{M}_{s} \sim M_{s}$ via the Green-Schwarz mechanism, i.e. $\left.M_{V}^{2} \equiv K_{C, C *}\right|_{V=0}$, and furthermore there is the charged modulus dependent Fayet-Iliopoulos D-term: $\left.\xi \equiv K_{C}\right|_{V=0}$. Note that for the sake of specificity we assumed that the VEV's of the uncharged moduli $U=\mathcal{O}(1)$, which leads to $M_{p l} \sim M_{s} \sim \tilde{M}_{s}$. Generalizations to lower string scales are straightforward, but they require a more careful treatment of $\tilde{M}_{s}$.

$W_{0}$ and $K_{0}$ are the low energy superpotential and Kähler potential, and $\tilde{W}$ and $\tilde{K}$ are contributions to the superpotential and Kähler potential from the "hidden sector", responsible for the stabilization of the uncharged moduli $U$. Again, we assume that contributions from $\tilde{K}$ and $\tilde{W}$ are at a high (string) scale $\tilde{M}_{s} \gg \mathrm{TeV}$ and are sufficient to stabilize the uncharged moduli $U$ and break supersymmetry without giving rise to a cosmological constant. In $K_{0}, W_{0}$ and the potential $V_{0}$, then, $U$ 's are not dynamical variables and are replaced with their non-zero VEV's. This above splitting of $W$ and $K$ is justified when $\langle C\rangle \ll\langle U\rangle$ and $\frac{\langle\Phi\rangle}{\tilde{M}_{s}} \ll\langle U\rangle$, which for $\Phi$ is justified since we expect such a chiral matter field to be associated with low energies, and the solution for $C$ is justified a posteriori by the self-consistency of the derived solution as follows.

We examine the possible existence of a consistent solution where $\operatorname{Re}(C)$ and $\Phi$ stabilize near zero VEV. Expanding the Kähler potential around zero VEV in Re $(C)$ and $\Phi$ and keeping only the quadratic terms, we write

$$
K_{0}=\frac{\tilde{M}_{s}^{2}}{2}\left(C+C^{*}+Q_{a} V\right)^{2}+d \Phi \Phi^{*}
$$

where $d$ is a dimensionless $O(1)$ parameter. Note again, that in the type IIa context $\operatorname{Re}(C)$ is a complex structure modulus which parametrizes the deviation away from the special Lagrangian cycle wrapped by the $D_{a}$ brane stack. In the type IIb context these moduli specify the size of the blow-up moduli for the $D_{a}$ brane stack wrapping an orbifold singularity. Note that this structure of the Kähler potential, which is a quadratic function of $2 \operatorname{Re}(C)=C+C^{*}$, is expanded around zero VEV for $2 \operatorname{Re}(C)$. This is specific to charged moduli associated with gauge $D_{a}$-brane sectors, and it should be contrasted with those of the gauge coupling modulus in the heterotic string context, which typically have a logarithmic dependence on the dilaton modulus. Studies of moduli stabilization and supersymmetry breaking with charged moduli in the latter context can therefore lead to different conclusions. See, e.g. [54], and references therein. Again, the mass and Fayet-Iliopoulos parameter associated with the anomalous $\mathrm{U}(1)_{a}$ symmetry are $\left.M_{V}^{2} \equiv K_{C C^{*}}\right|_{V=0}=\tilde{M}_{s}^{2}$ and $\left.\xi \equiv K_{C}\right|_{V=0}=\tilde{M}_{s}^{2}\left(C+C^{*}\right)$, respectively.

The effective low energy potential can now be written as a sum of the low energy $\mathcal{N}=1$ global scalar potential for $\Phi$ fields and their soft supersymmetry breaking terms (from the hidden sector $\tilde{W}(U)$ ), as well as a potential F-term contribution due to charged moduli $C$, and we write these contributions as $V_{0}$. Furthermore, there is the $D$-term contribution of the anomalous $\mathrm{U}(1)_{a}$, so that the scalar potential is of the form

$$
V=V_{0}(\Phi,\langle U\rangle, C)+\frac{g^{2}}{2} D^{2}=V_{0}(\Phi,\langle U\rangle, C)+\frac{g^{2}}{2} \tilde{M}_{s}^{4}\left(C+C^{*}-\frac{Q_{a}|\Phi|^{2}}{\tilde{M}_{s}^{2}}\right)^{2} .
$$


Minimizing this potential with respect to $C$, we see that

$$
0=\frac{\partial V}{\partial C}=\frac{\partial V_{0}}{\partial C}+g^{2} \tilde{M}_{s}^{4}\left(C+C^{*}-\frac{Q_{a}|\Phi|^{2}}{\tilde{M}_{s}^{2}}\right)
$$

which gives

$$
C+C^{*}=\frac{Q_{a}|\Phi|^{2}}{\tilde{M}_{s}^{2}}-\frac{1}{g^{2} \tilde{M}_{s}^{4}} \frac{\partial V_{0}}{\partial C}=O\left(\frac{\mathrm{TeV}^{2}}{\tilde{M}_{s}^{2}}\right)+O\left(\frac{\mathrm{TeV}^{4}}{\tilde{M}_{s}^{4}}\right) \approx 0
$$

and thus $C+C^{*}$ is stabilized very close to zero. Note that the second term is suppressed by $\mathrm{TeV}^{2} / \tilde{M}_{s}^{2}$ relative to the first and can be therefore be dropped. Integrating out the charged modulus and minimizing the potential with respect to $\Phi$, we obtain

$$
\begin{aligned}
\frac{\partial V}{\partial \Phi} & =\frac{\partial V_{0}}{\partial \Phi}+g^{2} \tilde{M}_{s}^{4}\left(\frac{-Q_{a} \Phi^{*}}{\tilde{M}_{s}^{2}}\right)\left(C+C^{*}-\frac{Q_{a}|\Phi|^{2}}{\tilde{M}_{s}^{2}}\right) \\
& =\frac{\partial V_{0}}{\partial \Phi}+g^{2} \tilde{M}_{s}^{4}\left(\frac{-Q_{a} \Phi^{*}}{\tilde{M}_{s}^{2}}\right)\left(-\frac{1}{g^{2} \tilde{M}_{s}^{4}} \frac{\partial V_{0}}{\partial C}\right) \sim O\left(\mathrm{TeV}^{3}\right)+O\left(\frac{\mathrm{TeV}^{5}}{\tilde{M}_{s}^{2}}\right) .
\end{aligned}
$$

Again, the second term is suppressed by a factor of $\mathrm{TeV}^{2} / \tilde{M}_{s}^{2}$ relative to the first. Moreover, minimization with respect to $C$ forces the $D$-term contribution to vanish, up to contributions of $O\left(\mathrm{TeV}^{8} / \tilde{M}_{s}^{4}\right)$. This is crucial, since by examining two terms in the $F$-term contributions to the scalar potential, we see

$$
V_{0}(\Phi,\langle T\rangle, C) \sim \frac{1}{K_{\Phi \Phi^{*}}}\left|D_{\Phi} W_{0}\right|^{2}+\frac{1}{K_{C C^{*}}}\left|D_{c} W_{0}\right|^{2} \sim O\left(\mathrm{TeV}^{4}\right)+O\left(\frac{\mathrm{TeV}^{6}}{\tilde{M}_{s}^{2}}\right) .
$$

Note again, that the D-term contribution to the scalar potential is suppressed by a factor of $\mathrm{TeV}^{4} / \tilde{M}_{s}^{4}$ relative to the first term in (B.9), and can therefore be dropped relative to $V_{0}$. Furthermore, from (B.9) we see that the second term (that involves F-terms due to charged moduli) is suppressed by a factor of $\mathrm{TeV}^{2} / \tilde{M}_{s}^{2}$ relative to the first, and it can also be dropped.

In conclusion, the real part of the charged modulus $C$ is stabilized near zero VEV due to the D-flatness constraint, or equivalently, integrating out the heavy vector super-multiplet enforces its $D$-term to effectively vanish. Moreover, terms involving $C$ in the $F$-term contribution to the scalar potential are highly suppressed, i.e. thus the only contribution to $V_{0}$ arises by replacing $\operatorname{Re}(C)$ by its zero VEV.

$$
V_{\mathrm{eff}}=V_{0}(\Phi,\langle U\rangle, \operatorname{Re}(C) \approx 0) .
$$

Thus, the dynamics of the charged matter fields $\Phi$ is determined by its $\mathcal{N}=1$ global potential, soft supersymmetry breaking terms terms arising from the hidden sector stabilization of the uncharged modulus $U$, and replacing the real part of $\operatorname{Re}(C)$ with near-zero VEV. Note, however, that the low energy potential can still depend on $\operatorname{Im}(C)$, due to soft supersymmetry breaking terms, and will typically receive a mass of the order of the $\mathrm{TeV}$ scale. 
Given the assumption of $U$ stabilization in a hidden sector which breaks supersymmetry and leaves zero cosmological constant, the stabilization scenario of the real part of the charged modulus is quite general. We point out that the analysis and conclusions spelled out above are closely related to those of [55]. We have, however, allowed for general soft supersymmetry breaking terms, which could lead to nonzero VEV's of charged matter fields $\Phi$ at the TeV scale. We have also carried out the study of the charged moduli decoupling in the presence of non-perturbative, charged modulus dependent superpotential couplings.

Open Access. This article is distributed under the terms of the Creative Commons Attribution Noncommercial License which permits any noncommercial use, distribution, and reproduction in any medium, provided the original author(s) and source are credited.

\section{References}

[1] R. Blumenhagen, M. Cvetič and T. Weigand, Spacetime instanton corrections in $4 D$ string vacua - The seesaw mechanism for D-brane models, Nucl. Phys. B 771 (2007) 113 [hep-th/0609191] [SPIRES].

[2] B. Florea, S. Kachru, J. McGreevy and N. Saulina, Stringy instantons and quiver gauge theories, JHEP 05 (2007) 024 [hep-th/0610003] [SPIRES].

[3] L.E. Ibáñez and A.M. Uranga, Neutrino Majorana masses from string theory instanton effects, JHEP 03 (2007) 052 [hep-th/0609213] [SPIRES].

[4] R. Blumenhagen, M. Cvetič, S. Kachru and T. Weigand, D-brane instantons in type II orientifolds, Ann. Rev. Nucl. Part. Sci. 59 (2009) 269 [arXiv:0902.3251] [SPIRES].

[5] P. Anastasopoulos, E. Kiritsis and A. Lionetto, On mass hierarchies in orientifold vacua, JHEP 08 (2009) 026 [arXiv:0905.3044] [SPIRES].

[6] M. Cvetič, J. Halverson and R. Richter, Mass Hierarchies from MSSM Orientifold Compactifications, JHEP 07 (2010) 005 [arXiv:0909.4292] [SPIRES].

[7] M. Cvetič, J. Halverson and R. Richter, Mass hierarchies vs. proton decay in MSSM orientifold compactifications, arXiv:0910.2239 [SPIRES].

[8] M. Cvetič and T. Weigand, Hierarchies from D-brane instantons in globally defined Calabi-Yau orientifolds, Phys. Rev. Lett. 100 (2008) 251601 [arXiv:0711.0209] [SPIRES].

[9] M. Cvetič and T. Weigand, A string theoretic model of gauge mediated supersymmetry beaking, arXiv:0807.3953 [SPIRES].

[10] S. Kachru, R. Kallosh, A.D. Linde and S.P. Trivedi, De Sitter vacua in string theory, Phys. Rev. D 68 (2003) 046005 [hep-th/0301240] [SPIRES].

[11] V. Balasubramanian, P. Berglund, J.P. Conlon and F. Quevedo, Systematics of moduli stabilisation in Calabi-Yau flux compactifications, JHEP 03 (2005) 007 [hep-th/0502058] [SPIRES].

[12] R. Blumenhagen, S. Moster and E. Plauschinn, Moduli stabilisation versus chirality for MSSM like type IIB orientifolds, JHEP 01 (2008) 058 [arXiv:0711.3389] [SPIRES].

[13] M. Cvetič, R. Richter, 2 and T. Weigand, Computation of D-brane instanton induced superpotential couplings - Majorana masses from string theory, Phys. Rev. D 76 (2007) 086002 [hep-th/0703028] [SPIRES]. 
[14] L.E. Ibáñez, A.N. Schellekens and A.M. Uranga, Instanton induced neutrino majorana masses in CFT orientifolds with MSSM-like spectra, JHEP 06 (2007) 011 [arXiv:0704.1079] [SPIRES].

[15] S. Antusch, L.E. Ibáñez and T. Macri, Neutrino masses and mixings from string theory instantons, JHEP 09 (2007) 087 [arXiv:0706.2132] [SPIRES].

[16] M. Cvetič and P. Langacker, D-instanton generated Dirac neutrino masses, Phys. Rev. D 78 (2008) 066012 [arXiv:0803.2876] [SPIRES].

[17] M. Cvetič, J. Halverson, P. Langacker and R. Richter, The Weinberg operator and a lower string scale in orientifold compactifications, arXiv:1001.3148 [SPIRES].

[18] J.R. Ellis, J.F. Gunion, H.E. Haber, L. Roszkowski and F. Zwirner, Higgs bosons in a nonminimal supersymmetric model, Phys. Rev. D 39 (1989) 844 [SPIRES].

[19] D. Suematsu and Y. Yamagishi, Radiative symmetry breaking in a supersymmetric model with an extra U(1), Int. J. Mod. Phys. A 10 (1995) 4521 [hep-ph/9411239] [SPIRES].

[20] M. Cvetič and P. Langacker, Implications of Abelian extended gauge structures from string models, Phys. Rev. D 54 (1996) 3570 [hep-ph/9511378] [SPIRES].

[21] C. Panagiotakopoulos and K. Tamvakis, New minimal extension of MSSM, Phys. Lett. B 469 (1999) 145 [hep-ph/9908351] [SPIRES].

[22] C. Panagiotakopoulos and A. Pilaftsis, Higgs scalars in the minimal non-minimal supersymmetric standard model, Phys. Rev. D 63 (2001) 055003 [hep-ph/0008268] [SPIRES].

[23] A. Dedes, C. Hugonie, S. Moretti and K. Tamvakis, Phenomenology of a new minimal supersymmetric extension of the standard model, Phys. Rev. D 63 (2001) 055009 [hep-ph/0009125] [SPIRES].

[24] A. Menon, D.E. Morrissey and C.E.M. Wagner, Electroweak baryogenesis and dark matter in the NMSSM, Phys. Rev. D 70 (2004) 035005 [hep-ph/0404184] [SPIRES].

[25] E. Accomando et al., Workshop on CP studies and non-standard Higgs physics, hep-ph/0608079 [SPIRES].

[26] V. Barger, P. Langacker and G. Shaughnessy, TeV physics and the Planck scale, New J. Phys. 9 (2007) 333 [hep-ph/0702001] [SPIRES].

[27] M. Maniatis, The next-to-minimal supersymmetric extension of the standard model reviewed, Int. J. Mod. Phys. A 25 (2010) 3505 [arXiv:0906. 0777] [SPIRES].

[28] U. Ellwanger, C. Hugonie and A.M. Teixeira, The next-to-minimal supersymmetric standard model, arXiv:0910.1785 [SPIRES].

[29] M. Chemtob and P. Hosteins, Searching singlet extensions of the supersymmetric standard model in $Z_{6-I I}$ orbifold compactification of heterotic string, Eur. Phys. J. C 68 (2010) 539 [arXiv: 0909.4497] [SPIRES].

[30] O. Lebedev and S. Ramos-Sanchez, The NMSSM and string theory, Phys. Lett. B 684 (2010) 48 [arXiv: 0912.0477] [SPIRES].

[31] G. Aldazabal, L.E. Ibáñez, F. Quevedo and A.M. Uranga, D-branes at singularities: a bottom-up approach to the string embedding of the standard model, JHEP 08 (2000) 002 [hep-th/0005067] [SPIRES]. 
[32] I. Antoniadis, E. Kiritsis and T.N. Tomaras, A D-brane alternative to unification, Phys. Lett. B 486 (2000) 186 [hep-ph/0004214] [SPIRES].

[33] I. Antoniadis, E. Kiritsis and T. Tomaras, D-brane standard model, Fortsch. Phys. 49 (2001) 573 [hep-th/0111269] [SPIRES].

[34] L.E. Ibáñez and R. Richter, Stringy instantons and Yukawa couplings in MSSM-like orientifold models, JHEP 03 (2009) 090 [arXiv:0811.1583] [SPIRES].

[35] G.K. Leontaris, Instanton induced charged fermion and neutrino masses in a minimal standard model scenario from intersecting D-branes, Int. J. Mod. Phys. A 24 (2009) 6035 [arXiv: 0903.3691] [SPIRES].

[36] M. Cvetič, J. Halverson and R. Richter, 2, Realistic Yukawa structures from orientifold compactifications, JHEP 12 (2009) 063 [arXiv:0905.3379] [SPIRES].

[37] E. Kiritsis, M. Lennek and B. Schellekens, SU(5) orientifolds, Yukawa couplings, stringy instantons and proton decay, Nucl. Phys. B 829 (2010) 298 [arXiv:0909.0271] [SPIRES].

[38] P. Anastasopoulos, G.K. Leontaris and N.D. Vlachos, Phenomenological analysis of D-brane Pati-Salam vacua, JHEP 05 (2010) 011 [arXiv: 1002.2937] [SPIRES].

[39] R. Blumenhagen, M. Cvetič, P. Langacker and G. Shiu, Toward realistic intersecting D-brane models, Ann. Rev. Nucl. Part. Sci. 55 (2005) 71 [hep-th/0502005] [SPIRES].

[40] R. Blumenhagen, B. Körs, D. Lüst and S. Stieberger, Four-dimensional string compactifications with D-branes, orientifolds and fluxes, Phys. Rept. 445 (2007) 1 [hep-th/0610327] [SPIRES].

[41] F. Marchesano, Progress in D-brane model building, Fortsch. Phys. 55 (2007) 491 [hep-th/0702094] [SPIRES].

[42] M. Cvetič, G. Shiu and A.M. Uranga, Chiral four-dimensional $N=1$ supersymmetric type IIA orientifolds from intersecting D6-branes, Nucl. Phys. B 615 (2001) 3 [hep-th/0107166].

[43] M. Cvetič, G. Shiu and A.M. Uranga, Three-family supersymmetric standard like models from intersecting brane worlds, Phys. Rev. Lett. 87 (2001) 201801 [hep-th/0107143] [SPIRES].

[44] R. Blumenhagen, M. Cvetič, D. Lüst, R. Richter, 2 and T. Weigand, Non-perturbative Yukawa couplings from string instantons, Phys. Rev. Lett. 100 (2008) 061602 [arXiv: 0707.1871] [SPIRES].

[45] S. Weinberg, Varieties of Baryon and Lepton Nonconservation, Phys. Rev. D 22 (1980) 1694 [SPIRES].

[46] P. Anastasopoulos, T.P.T. Dijkstra, E. Kiritsis and A.N. Schellekens, Orientifolds, hypercharge embeddings and the standard model, Nucl. Phys. B 759 (2006) 83 [hep-th/0605226] [SPIRES].

[47] J.E. Kim and H.P. Nilles, The $\mu$ problem and the strong CP problem, Phys. Lett. B 138 (1984) 150 [SPIRES].

[48] G.F. Giudice and A. Masiero, A natural solution to the $\mu$ problem in supergravity theories, Phys. Lett. B 206 (1988) 480 [SPIRES].

[49] M. Cvetič, D.A. Demir, J.R. Espinosa, L.L. Everett and P. Langacker, Electroweak breaking and the mu problem in supergravity models with an additional $\mathrm{U}(1)$,

Phys. Rev. D 56 (1997) 2861 [hep-ph/9703317] [SPIRES]. 
[50] S. Chang, R. Dermisek, J.F. Gunion and N. Weiner, Nonstandard Higgs boson decays, Ann. Rev. Nucl. Part. Sci. 58 (2008) 75 [arXiv:0801.4554] [SPIRES].

[51] V. Barger, P. Langacker, H.-S. Lee and G. Shaughnessy, Higgs sector in extensions of the MSSM, Phys. Rev. D 73 (2006) 115010 [hep-ph/0603247] [SPIRES].

[52] L.E. Ibáñez, F. Marchesano and R. Rabadán, Getting just the standard model at intersecting branes, JHEP 11 (2001) 002 [hep-th/0105155] [SPIRES].

[53] M. Cvetič, P. Langacker and J. Wang, Dynamical supersymmetry breaking in standard-like models with intersecting D6-branes, Phys. Rev. D 68 (2003) 046002 [hep-th/0303208] [SPIRES].

[54] E. Dudas, Y. Mambrini, S. Pokorski, A. Romagnoni and M. Trapletti, Gauge vs. Gravity mediation in models with anomalous U(1)'s, JHEP 03 (2009) 011 [arXiv:0809.5064] [SPIRES].

[55] M.P. Garcia del Moral, A new mechanism of Kähler moduli stabilization in type IIB theory, JHEP 04 (2006) 022 [hep-th/0506116] [SPIRES]. 